$13^{\text {th }}$ International Conference on

AEROSPACE SCIENCES \& AVIATION TECHNOLOGY,

ASAT- 13, May 26 - 28, 2009, E-Mail: asat@mtc.edu.eg

Military Technical College, Kobry Elkobbah, Cairo, Egypt

Tel : +(202) $24025292-24036138$, Fax: $+(202) 22621908$

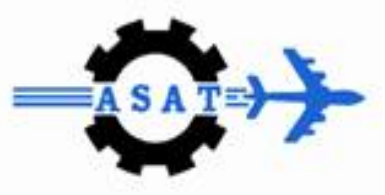

\title{
Dynamic Modeling of Robotics for Various Regions of Speed (DMRS)
}

\author{
Eyad Sh. Jabbour ${ }^{*}$, Hussein M. Mahgoub ${ }^{* *}$, Nabil G. Mekhail ${ }^{* *}$, Ahmed M. A. Elraouf ${ }^{* * *}$
}

\begin{abstract}
The existing heavy rigid manipulators are shown to be inefficient in terms of power consumption or speed with respect to the operating payload. Also, the operation of high precision robots is severely limited by their dynamic deflection, which delays subsequent operations, thus conflicting with the demand of increased productivity. These conflicting requirements between high speed and high accuracy have rendered the robotic assembly task a challenging research problem. The choice of modeling techniques and dynamic analysis based on the operating regions of robotic speed plays effective factor for economical computation and efficient performance for the robotic job in question. Consequently, the modeling of robots can be classified into three main domains, which are rigid linkage domain, Semi-elastic linkage domain and Flexible linkage robotics domain. A numerical case study is presented to investigate that concept.
\end{abstract}

Keywords: Dynamic modeling, Robot dynamics, Robot classification, rigid domain, flexible domain

\section{Introduction}

Research on the dynamic modeling and control of flexible manipulators has received increased attention since the last 35 years due to their several advantages over rigid ones[1]. Unlike rigid manipulators, the dynamics of this class of manipulators incorporate the effects of mechanical flexibilities in both the links and joints. Link flexibility is a consequence of the lightweight constructional feature in manipulator arms that are designed to operate at high speed with low inertia. Joint flexibility arises because of the elastic behavior of the joint transmition elements such as gears and shafts. Thus, generally speaking manipulators undergo two commonly known types of motion, i.e. rigid and flexible motion. Accurate dynamic models should include the mutual coupling terms between rigid-body motion (so called nominal motion of robot) and the elastic motion [2],[6].

Most of the existing robotic manipulators are designed and build in a manner to maximize stiffness in an attempt to minimize the vibration of the end-effector to achieve good position accuracy. This high stiffness is achieved by using heavy material and a bulky design. Hence, the existing heavy rigid manipulators are shown to be inefficient in terms of power consumption or speed with respect to the operating payload. Also, the operation of high precision robots is severely limited by their dynamic deflection, which persists for a period of time after a move is completed.

\footnotetext{
Syrian Armed Forces

** Egyptian Armed Forces

${ }^{* * *}$ Prof. Dr., Menoufia University
} 
The settling time required for this residual vibration delays subsequent operations, thus conflicting with the demand of increased productivity. These conflicting requirements between high speed and high accuracy have rendered the robotic assembly task a challenging research problem. Also, many industrial manipulators face the problem of arm vibrations during high speed motion. In order to improve industrial productivity, it is required to reduce the weight of the arms and/or to increase their speed of operation. For these purposes it may be desirable to build flexible robotic manipulators.

Compared to the conventional heavy and bulky robots, flexible link manipulators have the potential advantage of lower cost, larger work volume, higher operational speed, greater payload-to-manipulator-weight ratio, smaller actuators, lower energy consumption, better maneuverability, better transportability and safer operation due to reduced inertia. But the greatest disadvantage of these manipulators is the vibration problem due to low stiffness. Because of the interaction of these motions, the resulting dynamic equations of flexible manipulators are highly complex. In practical applications and economical computational effort, the choice of robotics' speed is a significant factor for an efficient performance of the particular robotic job.

In the case of semi elastic robotic domain, the kinematic and dynamic analysis are focused on the manipulators having large light flexible arm or the flexibility effects of certain link on the robotic performance and on the operating efficiency in the case of small elastic motion about large rigid motion of certain robotic link.

Industrial robots used in car factories with heavy weights and large dimensions are examples of robots working in rigid domain. Space robots required to weigh as small as possible are belongs to elastic robotic domain, while robots used in electronic industrial applications can be considered to classified under semi elastic robotic domain due to the combination between conflicting features such as high precision and high velocity related to productivity demand. In the present paper, the effect of varying robot speed was obtained with respect to rigid, semi rigid and flexible robot links.

\section{Energy Consideration of Robotic Model}

In view of modeling techniques the dominant effect of each component of either kinetic energy or potential energy depends mainly on the operating conditions of the manipulator robot in question. For nonnatural manipulator robot, the kinetic and potential energies can be partitioned in the following form respectively

$T=T_{m}+T_{p}+T_{r}$

$V=V_{m}+V_{p}+V_{G}$

In view of kinetic energy consideration, the first component $\boldsymbol{T}_{\boldsymbol{m}}$ for the case of high speed undamped robot to be partitioned in the following form

$T_{m}=\frac{1}{2} \dot{q}^{T} M \dot{q}+\dot{q}^{T} G q+\frac{1}{2} q^{T} H q$

The first part represents the kinetic energy associated with natural robotic model in the limit of law speed manipulator here as 


$$
T_{m}=\frac{1}{2} \dot{q}^{T} M \dot{q}
$$

where $M$ is symmetric mass matrix measured in terms of the generalized coordinate $n$ of the manipulator assembly $\left(q_{1}, q_{2} \ldots q_{n}\right)$

The second part is apparent dissipative energy producing forces of coriolis type, where $\mathrm{G}$ is known as skew symmetric gyroscopic matrix. The second part has dominant effects on dynamic analysis of high speed rotary manipulators.

The third part is apparent potential energy giving rise to the centrifugal force, where $\mathrm{H}$ is known as skew symmetric centripetal matrix. For the case of low speed rotary manipulator model, the third part can be ignored compared with the actual potential energy of the model.

The second part is concerned with the heavy duty manipulator provided with massive payload end-effector $m_{p}$ compared with the mass $m_{l}$ of the last link of the arm. However, for the case at which the mass ratio $m_{p} / m_{l}<0.1$, the kinetic energy of payload can be ignored in the modeling.

The third part represents the inertia effect of the motor driver of the joints on the dynamic behavior of the robotic model. When the dynamic analysis is focused on the manipulator model, the third part can be ignored with sufficient accuracy.

In view of potential energy for modeling robot assembly, the first term represents the strain energy of links of robot which have dominant effects on dynamic analysis of high speed robot (modeled in flexible domain) here as

$V_{m}=\frac{1}{2} q^{T}\left(K+K_{f}\right) q$

where $K$ represents symmetric stiffness matrix associated with flexibility of links, $K_{f}$ represents quasidiagonal stiffness matrix associated with the model of flexibility of joints, in the case of modeling the manipulator assembly in rigid domain or semielastic domain, the sub matrices of stiffness matrix $\mathrm{K}$ associated with rigid links ( characterized by high stiffness, short length link compared with other links of robot model) can be neglected. The second part represents the potential energy due to the gravity effect of the payload end effector assembly

In the case of light payload, the potential energy $V_{P}$ can be neglected with sufficient accuracy of the modeling. The third part concerned with the gravity energy of all links taken into account on the modeling technique. When the inertia distribution is considered, the associated apparent stiffness matrix can be derived either by utilizing F.E.M [8] in the case of flexible link or by using velocity distribution technique [7] in the case of rigid link. Otherwise the technique of center mass is utilized for the sake of simplicity [9].

\section{Two link planar dynamic model}

A two link planar robot is under concern, Fig. 1. ,for which the vector of generalized coordinates is $q=\left[\theta_{1} \theta_{2}\right]$. let $\mathrm{l}_{1} \mathrm{l}_{2}$ be the distance of the center of mass of the two links from the respective joint axes. Let $m_{l 1}, m_{l 2}$ be the masses of the two links, and $\mathrm{m}_{\mathrm{m} 2}$ the mass motor of the elbow joint, and $I_{l 1}, I_{l 2}$ the moments of inertia relative to the centers of mass of the two links, respectively. It is assumed that the motors are located on the joint axes with centers of mass located at the origins of the respective frames. 


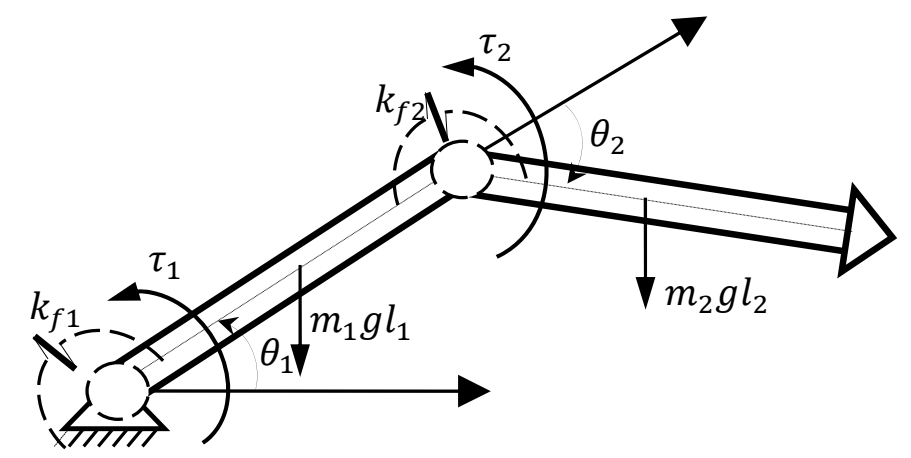

Fig. 1.Two Link Planar Arm with negligible mass of payload

By using Lagrange approach [3] and [4] the equations of motion for two rigid link robot can be obtained

The final mass matrix $\mathrm{M}$ is given by:

$M=\left[\begin{array}{ll}M_{1} & M_{2} \\ M_{3} & M_{4}\end{array}\right]$

where:

$M_{1}=\left[\begin{array}{ccc}I_{11} & 0 & 0 \\ 0 & m_{2} & 0 \\ 0 & 0 & m_{2}\end{array}\right]$

$I_{11}=\frac{1}{3} m_{1} l_{1}^{2}+m_{m 2} l_{1}^{2}+I_{m 2}$

$M_{2}=\left[\begin{array}{ccc}0 & l_{1} \sin \left(\theta_{1}\right) & -l_{1} \cos \left(\theta_{1}\right) \\ -\frac{1}{2} m_{2} l_{2} \sin \left(\theta_{1}+\theta_{2}\right) & 1 & 0 \\ \frac{1}{2} m_{2} l_{2} \cos \left(\theta_{1}+\theta_{2}\right) & 0 & 1\end{array}\right]$

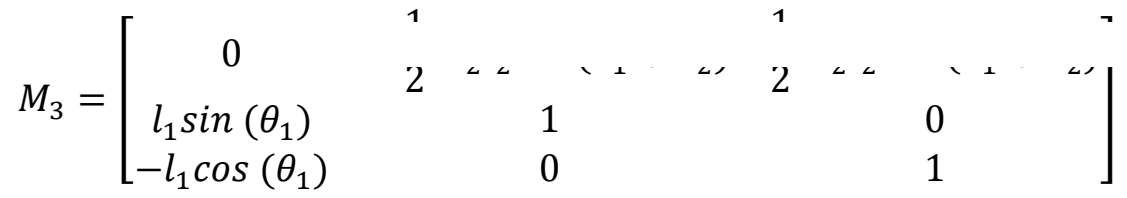

$M_{4}=\left[\begin{array}{ccc}\frac{1}{3} m_{2} l_{2}^{2} & 0 & 0 \\ 0 & 0 & 0 \\ 0 & 0 & 0\end{array}\right]$

The vector of generalized forces $Q$ : 


$$
Q=\left[\begin{array}{c}
-\frac{1}{2} m_{1} g l_{1} \cos \left(\theta_{1}\right)-m_{m 2} g l_{1} \cos \left(\theta_{1}\right)+\bar{\tau}_{1} \\
\frac{1}{2} m_{2} l_{2}\left(\dot{\theta}_{1}+\dot{\theta}_{2}\right)^{2} \cos \left(\theta_{1}+\theta_{2}\right) \\
\frac{1}{2} m_{2} l_{2}\left(\dot{\theta}_{1}+\dot{\theta}_{2}\right)^{2} \sin \left(\theta_{1}+\theta_{2}\right)-m_{2} g \\
-\frac{1}{2} m_{2} g l_{2} \cos \left(\theta_{1}+\theta_{2}\right)+\tau_{2} \\
-\dot{\theta}_{1}^{2} l_{1} \cos \left(\theta_{1}\right) \\
-\dot{\theta}_{1}^{2} l_{1} \sin \left(\theta_{1}\right)
\end{array}\right]
$$

The final equation of motion

$M \ddot{q}=Q$

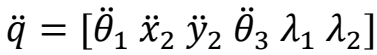

$\tau_{1}=\bar{\tau}_{1}+\tau_{2}+k_{f 1} \theta_{1}$

where:

$\bar{\tau}_{1}$ and $\tau_{2}$ are torques required to move link1 and link 2 respectively. $\lambda_{1}$ and $\lambda_{2}$ are Lagrange multipliers.

$$
\begin{gathered}
\lambda_{1}=\dot{\theta}_{1}^{2} m_{2} l_{1} \cos \theta_{1}+\ddot{\theta}_{1} m_{2} l_{1} \sin \theta_{1}+\left(\ddot{\theta}_{1}+\ddot{\theta}_{2}\right) \frac{m_{2} l_{2}}{2} \sin \left(\theta_{1}+\theta_{2}\right) \\
+\dot{\theta}_{3}^{2} \frac{m_{2} l_{2}}{2} \cos \left(\theta_{1}+\theta_{2}\right)
\end{gathered}
$$

$$
\begin{gathered}
\lambda_{2}=\dot{\theta}_{1}^{2} m_{2} l_{1} \sin \theta_{1}-\ddot{\theta}_{1} m_{2} l_{1} \cos \theta_{1}-\left(\ddot{\theta}_{1}+\ddot{\theta}_{2}\right) \frac{m_{2} l_{2}}{2} \cos \left(\theta_{1}+\theta_{2}\right) \\
+\dot{\theta}_{3}^{2} \frac{m_{2} l_{2}}{2} \sin \left(\theta_{1}+\theta_{2}\right)-m_{2} g
\end{gathered}
$$

$$
\begin{aligned}
\tau_{1}=\left\{\frac{1}{3} m_{1} l_{1}^{2}\right. & \left.+m_{m 2} l_{1}^{2}+I_{m 2}+I_{2}+m_{2}\left[l_{1}^{2}+\left(\frac{l_{2}}{2}\right)^{2}+l_{1} l_{2} \cos \theta_{2}\right]\right\} \ddot{\theta}_{1} \\
& +\frac{1}{2} m_{2} g l_{2} \cos \left(\theta_{1}+\theta_{2}\right)+\left\{m_{2}\left(\left(\frac{l_{2}}{2}\right)^{2}+\frac{1}{2} l_{1} l_{2} \cos \theta_{2}\right)+I_{2}\right\} \ddot{\theta}_{2} \\
& +h \dot{\theta}_{2}^{2}+2 h \dot{\theta}_{1} \dot{\theta}_{2}+k_{f 1} \theta_{1}+l_{1} g\left(\frac{m_{1}}{2}+m_{2}+m_{m 2}\right) \cos \theta_{1}
\end{aligned}
$$

$$
\begin{aligned}
\tau_{2}=\left(I_{2}+m_{2}\right. & \left.\left(\frac{l_{2}}{2}\right)^{2}+\frac{1}{2} l_{1} l_{2} c_{2}\right) \ddot{\theta}_{1}+\left(I_{2}+m_{2}\left(\frac{l_{2}}{2}\right)^{2}\right) \ddot{\theta}_{2}-h \dot{\theta}_{1}^{2}+k_{f 2} \theta_{2} \\
& +\frac{1}{2} m_{2} g l_{2} \cos \left(\theta_{1}+\theta_{2}\right)
\end{aligned}
$$

where $\mathrm{s}_{1}=\sin \theta_{1}, \mathrm{~s}_{3}=\sin \left(\theta_{1}+\theta_{2}\right) \mathrm{c}_{1}=\cos \theta_{1}, \mathrm{c}_{3}=\cos \left(\theta_{1}+\theta_{2}\right)$ and $h=-\frac{1}{2} l_{1} l_{2} m_{2} s_{2}$ 
Equations 14 and 15 are used first to calculate the inverse dynamics to obtain the torque vector required for both joint motors to follow the desired trajectory. Lagrange approach [4] is adopted to obtain the mass and stiffness matrices for flexible links. Each flexible link is modeled as one beam element with three degrees of freedom related to rigid body motion in addition to six other degrees related to flexibility. The general mass and stiffness matrices and force vector Q for a planar flexible link can be given as follows:

$$
M=\left[\begin{array}{ccccccccc}
m & 0 & \frac{-1}{2} m l s & \frac{1}{2} m c & \frac{-1}{2} m s & \frac{-1}{12} m l s & \frac{1}{2} m c & \frac{-1}{2} m s & \frac{1}{12} m l s \\
0 & m & \frac{1}{2} m l c & \frac{1}{2} m s & \frac{1}{2} m c & \frac{1}{12} m l c & \frac{1}{2} m s & \frac{1}{2} m c & \frac{-1}{12} m l c \\
\frac{-1}{2} m l s & \frac{1}{2} m l c & \frac{1}{3} m l^{2} & 0 & \frac{3}{20} m l & \frac{1}{30} m l^{2} & 0 & \frac{7}{20} m l & \frac{-1}{20} m l^{2} \\
\frac{1}{2} m c & \frac{1}{2} m s & 0 & \frac{1}{3} m & 0 & 0 & \frac{1}{6} m & 0 & 0 \\
\frac{-1}{2} m s & \frac{1}{2} m c & \frac{3}{20} m l & 0 & \frac{13}{35} m & \frac{11}{210} m l & 0 & \frac{9}{70} m & \frac{-13}{420} m l \\
\frac{-1}{12} m l s & \frac{1}{12} m l c & \frac{1}{30} m l^{2} & 0 & \frac{11}{210} m l & \frac{1}{105} m l^{2} & 0 & \frac{13}{420} m l & \frac{-1}{140} m l^{2} \\
\frac{1}{2} m c & \frac{1}{2} m s & 0 & \frac{1}{6} m & 0 & 0 & \frac{1}{3} m & 0 & 0 \\
\frac{-1}{2} m s & \frac{1}{2} m c & \frac{7}{20} m l & 0 & \frac{9}{70} m & \frac{13}{420} m l & 0 & \frac{13}{35} m & \frac{-11}{210} m l \\
\frac{1}{12} m l s & \frac{-1}{12} m l c & \frac{-1}{20} m l^{2} & 0 & \frac{-13}{420} m l & \frac{-1}{140} m l^{2} & 0 & \frac{-11}{210} m l & \frac{1}{105} m l^{2}
\end{array}\right]
$$

The stiffness matrix K:

$$
K=\left[\begin{array}{ccccccccc}
0 & 0 & 0 & 0 & 0 & 0 & 0 & 0 & 0 \\
0 & 0 & 0 & 0 & 0 & 0 & 0 & 0 & 0 \\
0 & 0 & k_{f} & 0 & 0 & 0 & 0 & 0 & 0 \\
0 & 0 & 0 & \frac{E A}{l} & 0 & 0 & \frac{-E A}{l} & 0 & 0 \\
0 & 0 & 0 & 0 & { }_{12} \frac{E I}{l^{3}} & { }^{6} \frac{E I}{l^{2}} & 0 & -12 \frac{E I}{l^{3}} & { }^{6} \frac{E I}{l^{2}} \\
0 & 0 & 0 & 0 & 6 \frac{E I}{l^{2}} & { }_{4} \frac{E I}{l} & 0 & -6 \frac{E I}{l^{2}} & { }_{2} \frac{E I}{l} \\
0 & 0 & 0 & \frac{-E A}{l} & 0 & 0 & \frac{E A}{l} & 0 & 0 \\
0 & 0 & 0 & 0 & -12 \frac{E I}{l^{3}} & -6 \frac{E I}{l^{2}} & 0 & \frac{12}{l^{3}} & { }^{6} \frac{E I}{l^{2}} \\
0 & 0 & 0 & 0 & \frac{6}{l^{2}} & \frac{2}{l} & 0 & -6 \frac{E I}{l^{2}} & { }_{4} \frac{E I}{l}
\end{array}\right]
$$

The vector of generalized forces Q:

$$
Q=\left[\begin{array}{lllllllll}
\frac{1}{2} \dot{\theta}^{2} m l c & \frac{1}{2} \dot{\theta}^{2} m l s & 0 & \frac{1}{6} \dot{\theta}^{2} m l & 0 & 0 & \frac{1}{3} \dot{\theta}^{2} m l & 0 & 0
\end{array}\right]^{T}
$$


Where $E, A, I, l, m, k_{f}, \dot{\theta}$ are modulus of elasticity, area cross section, mass moment of inertia, beam length, mass of the link, joint stiffness and angular velocity respectively. $\mathrm{s}=\sin \theta, \mathrm{c}=\cos \theta$.

The M, K and Q matrices are utilized to build the first rigid-second flexible $(R-F)$ and first flexible-second flexible (F-F) robot models by using standard Finite Element method.

\section{Case study}

By considering the two link planar robot in Fig.1, The properties for both links are tabulated in Table 1 , it is desired for both links to follow the same joint trajectories. Each of The two joints has to pass $\frac{\pi}{2} \mathrm{rad}$ as total variation during $0.6 \mathrm{sec}$ period of time for three cases different from each other in maximum operating velocity $\mathrm{Vm}$. Three values of $\mathrm{Vm}$ are chosen to generate their related trajectories, which are $1.1 \pi, 1.3 \pi$ and $1.5 \pi$.

\section{Table 1. Links Properties}

\begin{tabular}{|l|c|l|}
\hline $\mathrm{E} 1, \mathrm{E} 2$ & $2 \mathrm{e} 11 \mathrm{~N} / \mathrm{m}^{2}$ & Young modulus \\
\hline $\mathrm{I} 1, \mathrm{I} 2$ & $9.0000 \mathrm{e}-011 \mathrm{~m}^{4}$ & Area moments of inertia \\
\hline$\rho 1, \rho 2$ & $7800 \mathrm{~kg} / \mathrm{m}^{3}$ & Mass density \\
\hline $\mathrm{a} 1, \mathrm{a} 2$ & $0.003 * .04 \mathrm{~m}^{2}$ & Cross-sectional areas \\
\hline $\mathrm{L} 1, \mathrm{~L} 2$ & $0.5 \mathrm{~m}$ & Length of the two links \\
\hline$m_{m 2}$ & $0.12 \mathrm{~kg}$ & Mass of Motor 2 \\
\hline$k_{f 1,} k_{f 2}$ & $0.1 \mathrm{~N} . \mathrm{m} / \mathrm{rad}$ & stiffness of Joint 1 and 2 \\
\hline
\end{tabular}

\subsection{Joint Space Trajectory}

The trapezoidal velocity profile is assigned, which imposes a constant acceleration in the start phase, a cruise velocity, and a constant deceleration in the arrival phase. The resulting trajectory is formed by a linear segment connected by two parabolic segments to the initial and final positions (trapezoidal velocity profiles [2]).it is assumed that both initial and final velocities are null and the segments with constant acceleration has the same time duration. The velocity at the end of the parabolic segment must be equal to the constant velocity of the linear segment.

$$
q(t)=\left\{\begin{array}{cc}
q_{i}+\frac{1}{2} \ddot{q}_{c} t^{2} & 0 \leq t \ll t_{c} \\
q_{i}+\ddot{q}_{c} t_{c}\left(t-t_{c} / 2\right) & t_{c}<t \ll t_{f}-t_{c} \\
q_{f}-\frac{1}{2} \ddot{q}_{c}\left(t_{f}-t\right)^{2} & t_{f}-t_{c}<t \ll t_{f}
\end{array}\right\}
$$

where:

$q_{i}$ and $q_{f}$ are initial and final joint displacements respectively.

$t_{c}$ is the time at the end of the first parabolic segment.

$q_{c}$ is the joint displacement at time $t_{c}$.

$t_{f}$ is the time at the end point of trajectory. 
The joint space trajectories related to these three maximum velocities ore obtained by applying the trapezoidal velocity approach. Fig. 2. and Fig. 3. Represent the displacements and velocities which are correspondent to these three velocities.

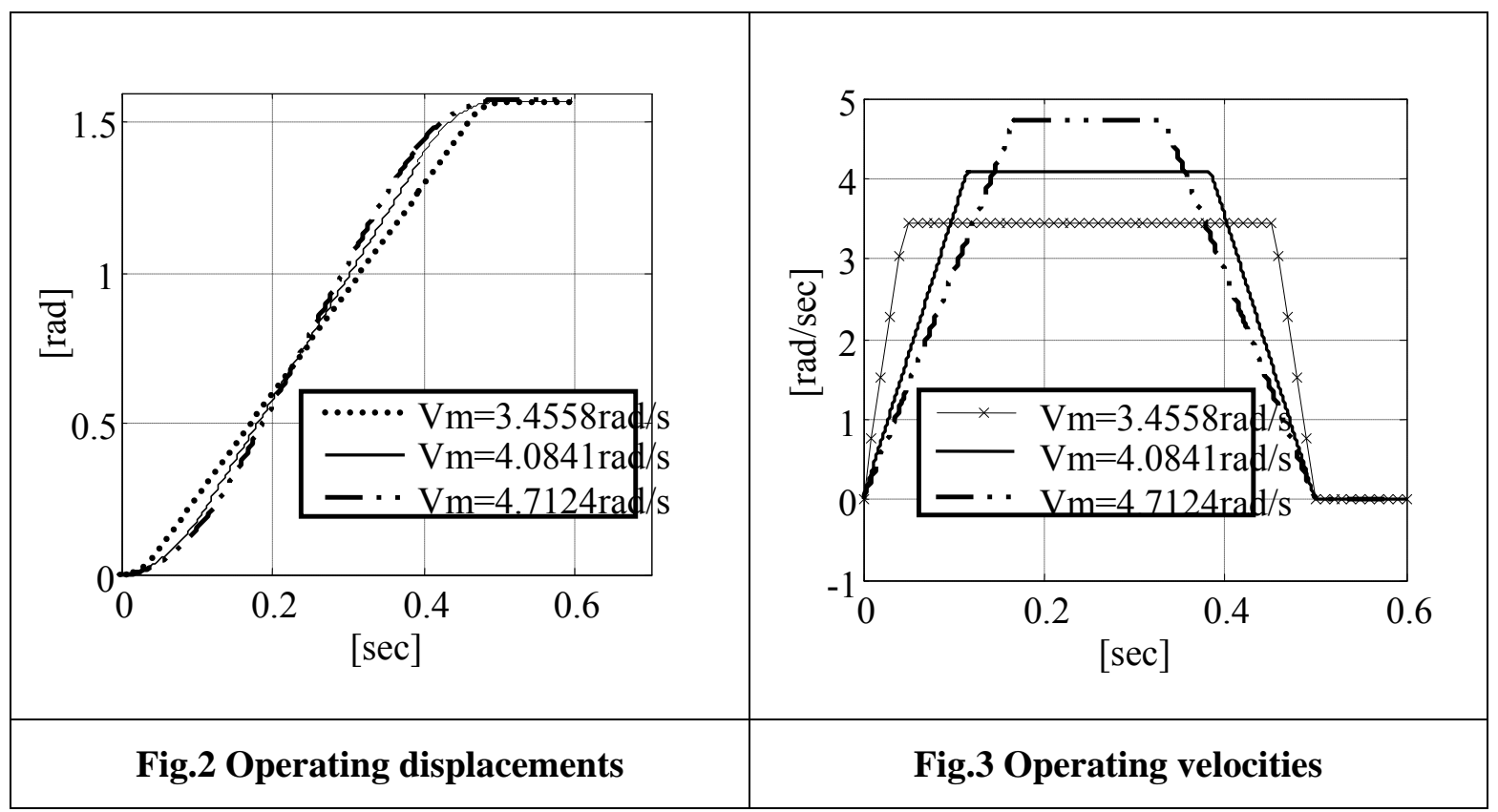

Now by using digital values of vectors $q, \dot{q}$ and $\ddot{q}$ obtained previously for the three trajectories, Equations (14) and (15) can be utilized to calculate the required torque for each joint associated to the three cases (doing the inverse dynamics). Fig. 4. and Fig. 5 show the torques required for the robot joints to track the trajectory for $V m=3.4558 \frac{\mathrm{rad}}{\mathrm{sec}}$ while Figs. (6 $\& 7)$ and $(8 \& 9)$ show the torques required for the robot joints to track the trajectory for $V m=4.0841 \frac{\mathrm{rad}}{\mathrm{sec}}$ and $V m=4.7124 \frac{\mathrm{rad}}{\mathrm{sec}}$ respectively. The difference between velocities undergoes the trapezoidal velocity approach constrains and choosing them in a small range proves the model sensitivity to velocity variation.

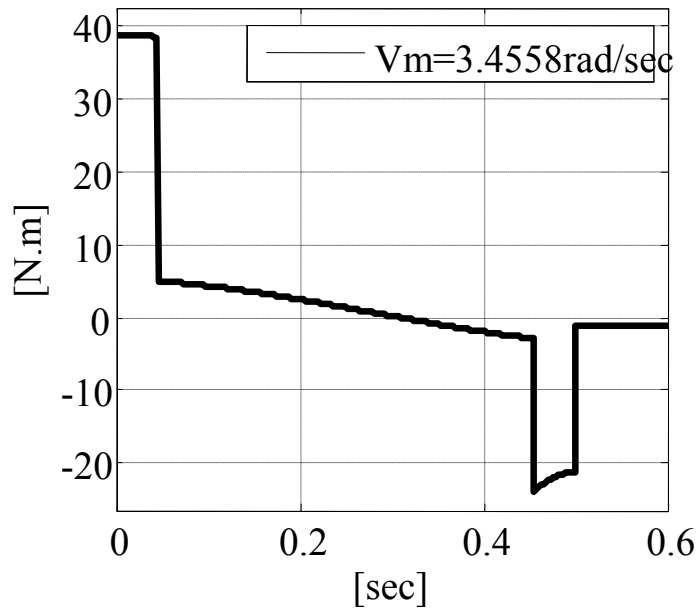

Fig.4. Joint 1 torque

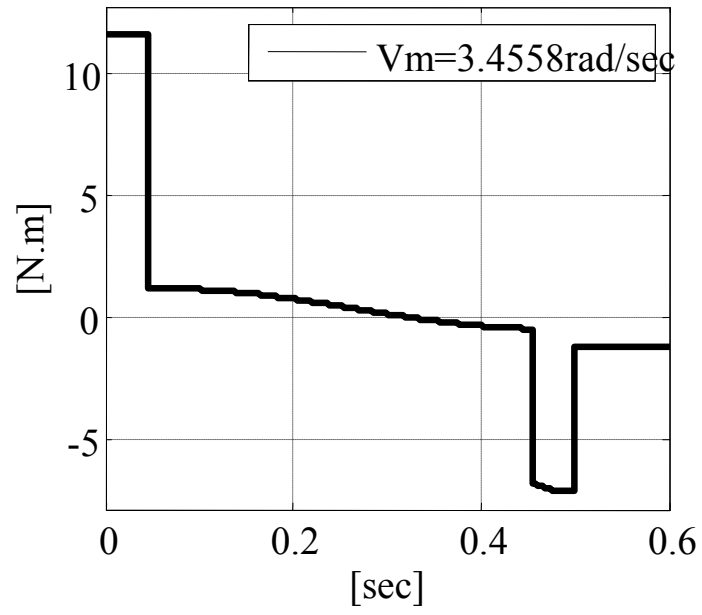

Fig.5. Joint 2 torque 


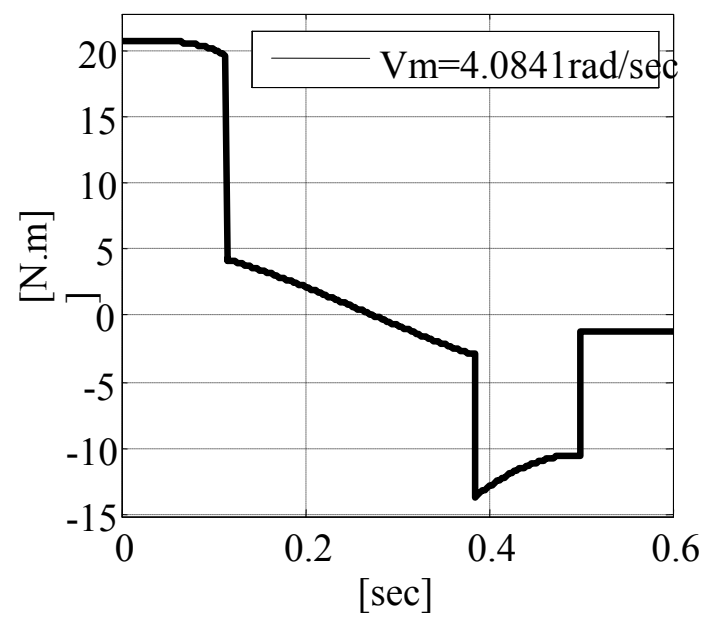

Fig.6. Joint 1 torque

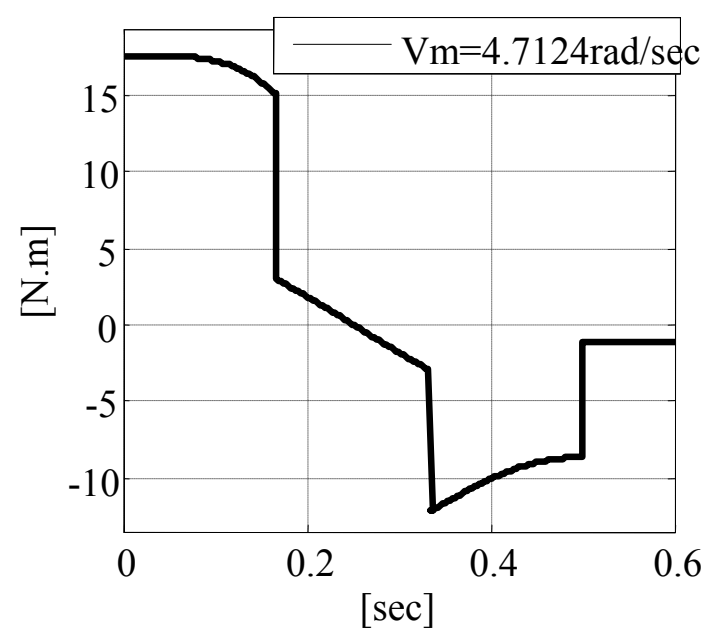

Fig.8. Joint 1 torque

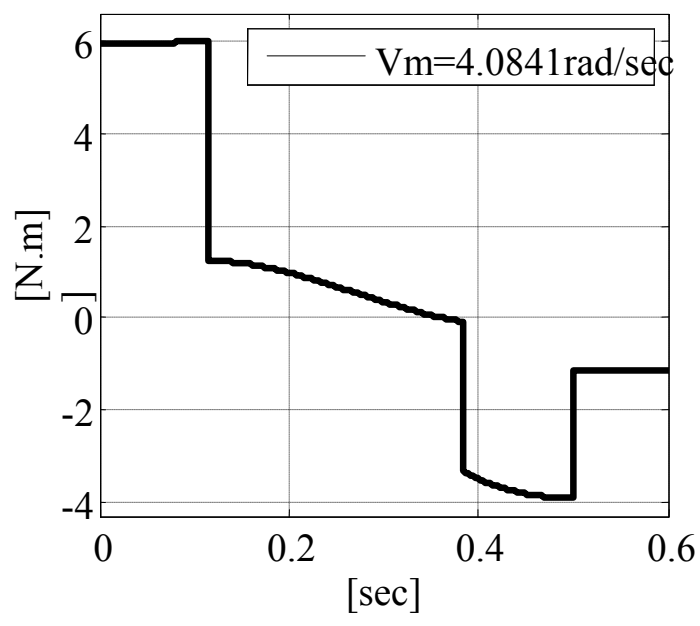

Fig.7. Joint 2 torque

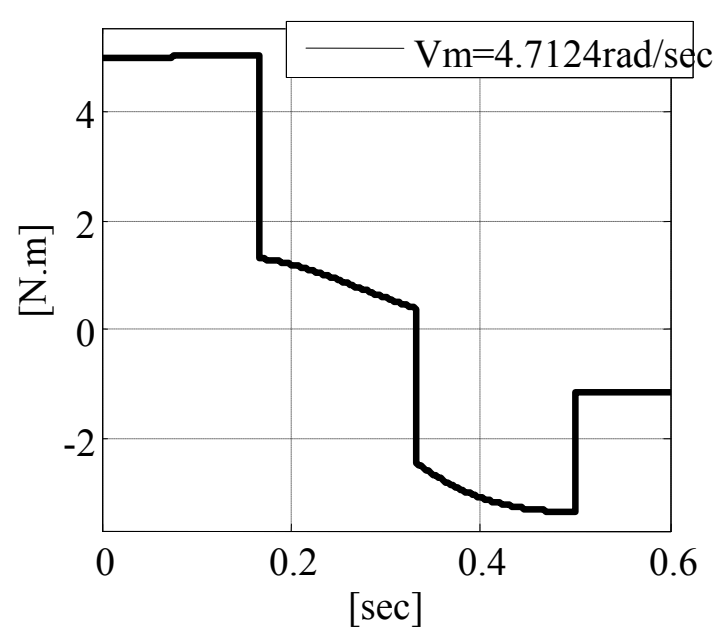

Fig.9. Joint 2 torque

After getting the torque vectors required for joint actuators to move the two links of robot according to the trajectories assigned before, It is possible now to use them as input data to the three models which are first rigid-second rigid (R-R), first rigid-second flexible (R-F) and first flexible-second flexible (F-F).

The flexibility will take its effect on the output joint space trajectories. The results from three models are compared for each of the three chosen maximum velocity to obtain the curves in Figures from 11 to 16.where the odd curve number related to joint 1 while the even one related to joint 2 each time. In the following figures $e_{R R}, e_{R F}$ and $e_{F F}$ represents errors for rigid, semi flexible and flexible domains respectively. 
where $\mathrm{e}=\left|\frac{\text { calculated value-reference value }}{\text { reference value }}\right| \%$

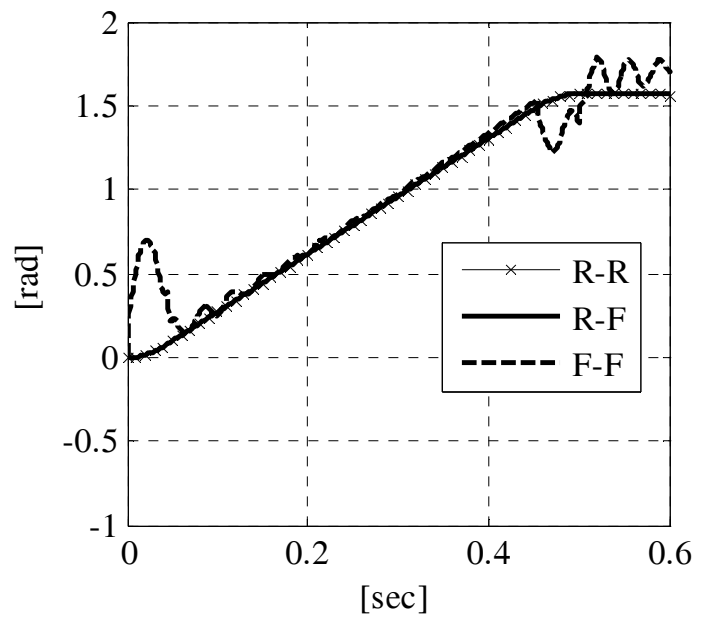

Fig.11. Joint 1 angle( $(\mathrm{Vm}=3.4558 \mathrm{rad} / \mathrm{sec})$

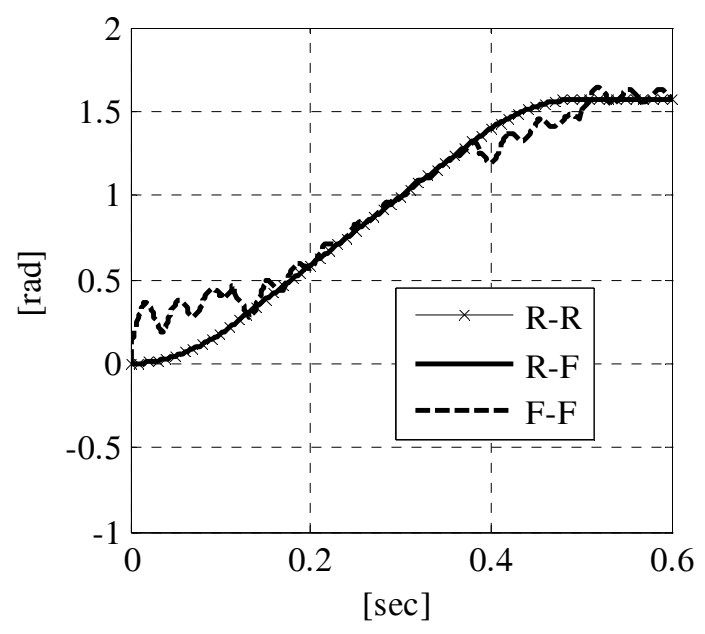

Fig.13. Joint 1 angle(Vm=4.0841rad/sec)

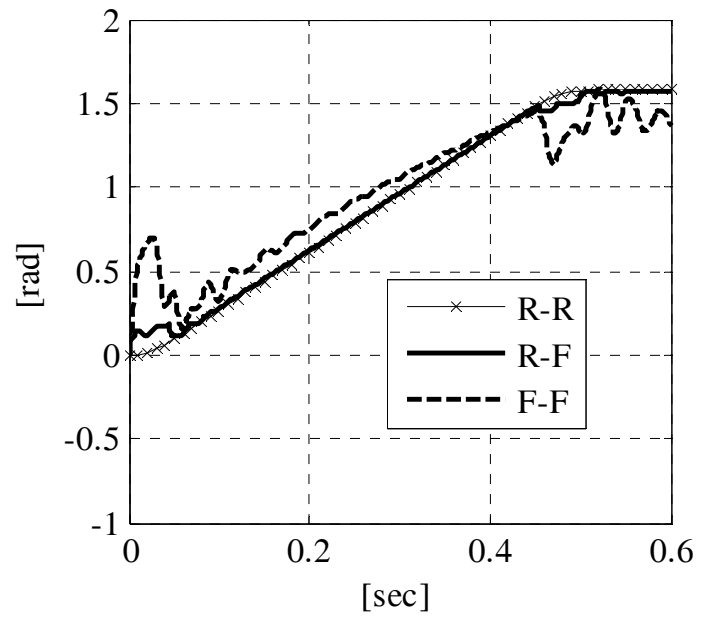

Fig.12. Joint 2

. ... -... . .

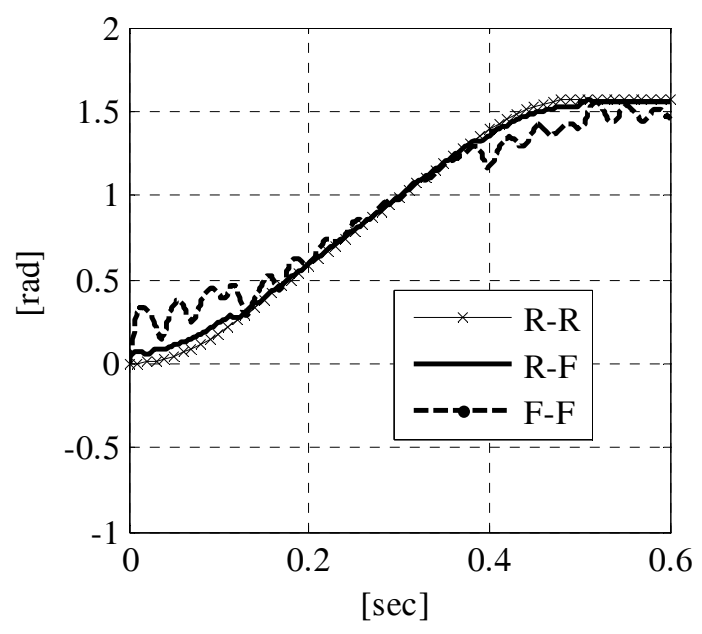

Fig.14. Joint 2 


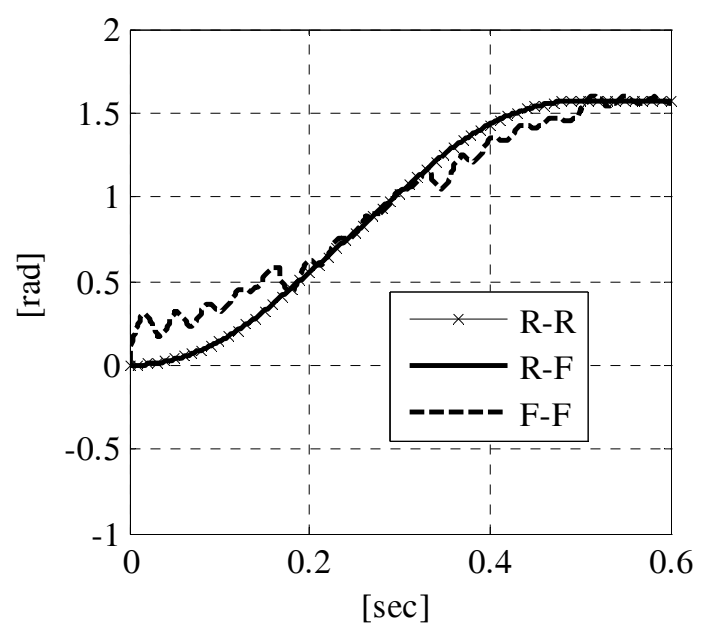

Fig.15. Joint 1 angle(Vm=4.7124rad/sec)

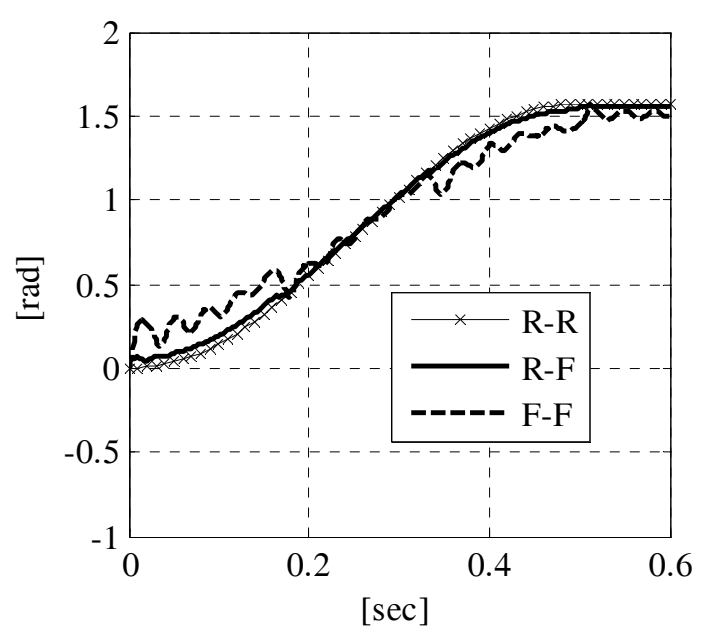

Fig.16. Joint 2

\section{Conclusion}

Moving the robot links in high assigned velocity, while offering extra time to reach this high velocity, is better than working with low maximum velocity with smaller available period of time, when flexibility is under concern. In fact, the rate of velocity is of significant important than the magnitude of velocity itself. Robots can be classified from flexibility point of view into three main groups which are rigid linkage, Semi-elastic linkage (mixed) and Flexible linkage robotics and when it is desired to utilize the benefits of lightweight constructional feature in manipulator arms while maintain acceptable response, it is recommended to shelter to the second group as can be found obviously from results depicted in figures 11 to 16 with an improvement of approximately $6 \%$ to $33 \%$ comparing to the third group.

\section{References}

[1] Alberto Trevisani and Maria Elena Valcher., "An Energy-Based Adaptive Control Design Technique for Multibody-Mechanisms With Flexible Links", "IEEE/ASME Transactions On Mechatronics", 2005, pp. 571-581.

[2] M. Giovagnoni, "A Numerical And Experimental Analysis Of A Chain Of Flexible Bodies", “ASME J. Dyna. Syst., Meas., Control”, 1994, pp. 73-80,

[3] Lorenzo Sciavicco and Bruno Siciliano, Modeling and Control of Robot Manipulator, McGraw-Hill Companies, US, 1996, pp.173-175.

[4] Low, K. H., and Vidyasagar, M., "A Lagrangian Formulation Of The Dynamic Model For Flexible Manipulator Systems," Journal of Dynamic Systems, Measurement and Control, 1988, pp. 175-181.

[5] Ashitava. G., Robotics Fundamental Concepts and Analysis, Oxford University, 2007, pp. 187-202.

[6] A. Maher, P.G. Kessel and R.D Cook, "A Partitioned Finite Element Method For Dynamical Systems"," journal computers and structures", 1984, pp. 81-91.

[7] A. Maher, Kinetic Analysis Of Large Nominal Motion Of Robots Using Velocity Distribution Approach", "journal of mechanisms". 